\title{
A Ranking Method of Triangular Intuitionistic Fuzzy Numbers and Application to Decision Making
}

\author{
Deng Feng Li * \\ School of Management, Fuzhou University \\ Fuzhou 350108, Fujian, China \\ E-mail: dengfengli@sina.com or lidengfeng@fzu.edu.cn
}

Jiang Xia Nan

College of Information Engineering, Dalian University

Dalian 116622, Liaoning, China

E-mail: jiangxia1107@163.com

Mao Jun Zhang

Department of Economics, Dalian University of Technology

Dalian 116024, Liaoning, China

E-mail: zmj@dlut.edu.cn

Received: 14-09-2009; Accepted: 22-04-2010

\begin{abstract}
Ranking of triangular intuitionistic fuzzy numbers (TIFNs) is an important problem, which is solved by the value and ambiguity based ranking method developed in this paper. Firstly, the concept of TIFNs is introduced. Arithmetic operations and cut sets over TIFNs are investigated. Then, the values and ambiguities of the membership degree and the non-membership degree for TIFNs are defined as well as the value-index and ambiguity-index. Finally, a value and ambiguity based ranking method is developed and applied to solve multiattribute decision making problems in which the ratings of alternatives on attributes are expressed using TIFNs. A numerical example is examined to demonstrate the implementation process and applicability of the method proposed in this paper. Furthermore, comparison analysis of the proposed method is conducted to show its advantages over other similar methods.
\end{abstract}

Keywords: Triangular intuitionistic fuzzy number, intuitionistic fuzzy set, ranking of triangular intuitionistic fuzzy numbers, multiattribute decision making.

\section{Introduction}

The ranking of fuzzy numbers is important in fuzzy multiattribute decision making (MADM). There exists a large amount of literature involving the ranking of fuzzy numbers ${ }^{1-9}$. Roughly speaking, a fuzzy number may be considered as a representation for an ill-known quantity. The intuitionistic fuzzy (IF) set introduced by Atanassov ${ }^{10}$ is a generalization of the fuzzy set ${ }^{11}$ and the IF set may express and describe information more abundant and flexible than the fuzzy set when uncertain information is involved. Therefore, an ill-known quantity may also be expressed with an intuitionistic fuzzy number (IFN) in the sense of Atanassov. Recently, the IFN receives little attention and different definitions of IFNs have been proposed as well as the corresponding ranking methods of IFNs. Mitchell ${ }^{12}$ interpreted an IFN as an ensemble of ordinary fuzzy numbers and introduced a ranking method. Nayagam et $\mathrm{al}^{13}$ described a type of IFNs and introduced a method

*Corresponding address: School of Management, Fuzhou University, No. 523, Industry Road, Fuzhou 350108, Fujian, China. Tel.: +86-059183768427. 
of IF scoring that generalized Chen and Hwang's scoring ${ }^{4}$ for ranking of IFNs. However, these existing definitions of IFNs are complicated and the ranking methods of IFNs have tedious calculations. By adding a degree of non-membership, Shu et al ${ }^{14}$ defined triangular intuitionistic fuzzy numbers (TIFNs), but not given the ranking of TIFNs. By an analogy, Wang and Zhang ${ }^{15}$ defined a trapezoidal IF number and its expected value as well as a ranking method. Wang and Zhang transformed the ranking of trapezoidal IF numbers to that of interval numbers. As far as we know, the ranking of interval numbers is a difficult problem. Furthermore, different ranking methods of interval numbers maybe produce different ranking results for TIFNs, which can bring some difficulties for decision makers. TIFNs are special trapezoidal IF numbers and are commonly used in MADM problems. However, there exists little investigation on the ranking of TIFNs. Nan et al ${ }^{16}$ defined the ranking order relations of TIFNs, which are applied to matrix games with payoffs of TIFNs. In this paper, a value and ambiguity based ranking method is developed for TIFNs. The proposed method transforms the ranking of TIFNs to that of real numbers, which is easy to be handled and calculated. Moreover, the proposed ranking method can be extended to that of trapezoidal IF numbers ${ }^{15}$.

There are always uncertainty and imprecision existing in real-life decision making information. In order to develop a good methodology, the fuzzy set ${ }^{17}$, linguisticvalued ${ }^{18}$ and IF set ${ }^{19-21}$ are frequently used to describe imprecise and uncertain factors appearing in real-life decision problems. In this paper, the concept of an TIFN is considered as a representation for these uncertain factors in real-life decision situations and we study MADM problems in which the ratings of alternatives on attributes are expressed using TIFNs.

The rest of this paper is organized as follows. In Section 2, the concept of an TIFN is introduced. The arithmetic operations and cut sets of TIFNs are given. Section 3 defines the concepts of the value and ambiguity of the membership and the non-membership degrees as well as the value-index and ambiguity-index. Furthermore, a new ranking method of TIFNs is developed on the value-index and ambiguity-index. Section 4 presents MADM problems in which the ratings of alternatives on attributes are expressed with TIFNs, which is solved by the extended additive weighted method using the value and ambiguity based ranking method proposed in this paper. A numerical example and short concluding remark are given in Sections 5 and 6, respectively.

\section{Basic Definitions}

\subsection{The definition and operations of TIFNs}

In this section, TIFNs and their operations are defined as follows.

Definition 1. An TIFN $\tilde{a}=<(\underline{a}, a, \bar{a}) ; w_{\tilde{a}}, u_{\tilde{a}}>$ is $a$ special IF set on the real number set $\mathrm{R}$, whose membership function and non-membership function are defined as in Fig. 1 as follows:

$$
\mu_{\tilde{a}}(x)=\left\{\begin{array}{cll}
w_{\tilde{a}}(x-\underline{a}) /(a-\underline{a}) & \text { if } & \underline{a} \leq x<a \\
w_{\tilde{a}} & \text { if } & x=a \\
w_{\tilde{a}}(\bar{a}-x) /(\bar{a}-a) & \text { if } & a<x \leq \bar{a} \\
0 & \text { if } & x<\underline{a} \text { or } x>\bar{a}
\end{array}\right.
$$

and

$$
v_{\tilde{a}}(x)=\left\{\begin{array}{cl}
{\left[a-x+u_{\tilde{a}}(x-\underline{a})\right] /(a-\underline{a})} & \text { if } \underline{a} \leq x<a \\
u_{\tilde{a}} & \text { if } x=a \\
{\left[x-a+u_{\tilde{a}}(\bar{a}-x)\right] /(\bar{a}-a)} & \text { if } a<x \leq \bar{a} \\
1 & \text { if } x<\underline{a} \text { or } x>\bar{a}
\end{array}\right.
$$

respectively, where the values $w_{\tilde{a}}$ and $u_{\tilde{a}}$ represent the maximum degree of membership and the minimum degree of non-membership, respectively, such that they satisfy the following conditions:

$$
0 \leq w_{\tilde{a}} \leq 1,0 \leq u_{\tilde{a}} \leq 1,0 \leq w_{\tilde{a}}+u_{\tilde{a}} \leq 1 .
$$

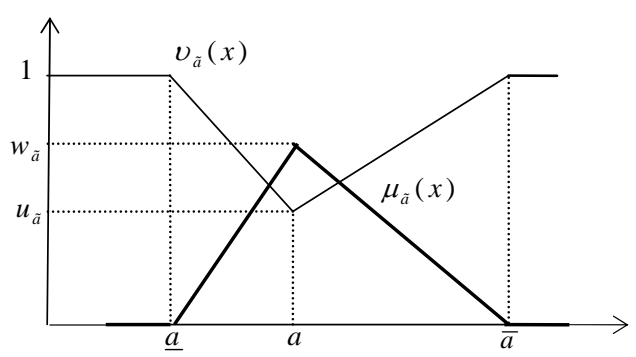

Fig. 1. An TIFN

Let

$$
\pi_{\tilde{a}}(x)=1-\mu_{\tilde{a}}(x)-v_{\tilde{a}}(x),
$$


which is called as the IF index of an element $x$ in $\tilde{a}$. It is the degree of indeterminacy membership of the element $x$ to $\tilde{a}$.

If $\underline{a} \geq 0$ and one of the three values $\underline{a}, a$ and $\bar{a}$ is not equal to 0 , then the TIFN $\tilde{a}=<(\underline{a}, a, \bar{a}) ; w_{\tilde{a}}, u_{\tilde{a}}>$ is called as a positive TIFN, denoted by $\tilde{a}>0$. Likewise, if $\bar{a} \leq 0$ and one of the three values $\underline{a}, a$ and $\bar{a}$ is not equal to 0 , then the TIFN $\tilde{a}=<(\underline{a}, a, \bar{a}) ; w_{\tilde{a}}, u_{\tilde{a}}>$ is called as a negative TIFN, denoted by $\tilde{a}<0$.

An TIFN $\tilde{a}=<(\underline{a}, a, \bar{a}) ; w_{\tilde{a}}, u_{\tilde{a}}>$ may express an illknown quantify, which is approximately equal to $a$. Namely, the ill-known quantify is expressed using any value between $\underline{a}$ and $\bar{a}$ with different degree of membership and degree of non-membership. In other words, the most possible value is $a$ with the degree of membership $w_{\tilde{a}}$ and the degree of non-membership $u_{\tilde{a}}$; the pessimistic value is $\underline{a}$ with the degree of membership 0 and the degree of non-membership 1; the optimistic value is $\bar{a}$ with the degree of membership 0 and the degree of non-membership 1 ; other value is any $x$ in the open interval $(\underline{a}, \bar{a})$ with the membership degree $\mu_{\tilde{a}}(x)$ and the non-membership degree $v_{\tilde{a}}(x)$.

It is easy to see that $\mu_{\tilde{a}}(x)+v_{\tilde{a}}(x)=1$ for any $x \in \mathrm{R}$ if $w_{\tilde{a}}=1$ and $u_{\tilde{a}}=0$. Hence, the TIFN $\tilde{a}=<(\underline{a}, a, \bar{a}) ; w_{\tilde{a}}, u_{\tilde{a}}>\quad$ degenerates to $\tilde{a}=<(\underline{a}, a, \bar{a}) ; 1,0>$, which is just about a triangular fuzzy number (TFN) ${ }^{22}$. Therefore, the concept of the TIFN is a generalization of that of the TFN ${ }^{22}$.

Two parameters $w_{\tilde{a}}$ and $u_{\tilde{a}}$ are introduced in Definition 1 to reflect the confidence level and nonconfidence level of the TIFN $\tilde{a}=<(\underline{a}, a, \bar{a}) ; w_{\tilde{a}}, u_{\tilde{a}}>$, respectively. Compared with the TFNs, TIFNs may express more uncertainty.

In a similar way to the arithmetic operations of the TFNs ${ }^{22}$, the arithmetic operations of TIFNs may be defined as follows ${ }^{23}$.

Definition 2. Let $\quad \tilde{a}=<(\underline{a}, a, \bar{a}) ; w_{\tilde{a}}, u_{\tilde{a}}>\quad$ and $\tilde{b}=<(\underline{b}, b, \bar{b}) ; w_{\tilde{b}}, u_{\tilde{b}}>$ be two TIFNs with $w_{\tilde{a}} \neq w_{\tilde{b}}$ and $u_{\tilde{a}} \neq u_{\tilde{b}} . \lambda$ is any real number. The arithmetic operations over TIFNs are defined as follows:

$$
\begin{aligned}
\tilde{a}+\tilde{b} & =<(\underline{a}+\underline{b}, a+b, \bar{a}+\bar{b}) ; w_{\tilde{a}} \wedge w_{\tilde{b}}, u_{\tilde{a}} \vee u_{\tilde{b}}> \\
\tilde{a}-\tilde{b} & =<(\underline{a}-\bar{b}, a-b, \bar{a}-\underline{b}) ; w_{\tilde{a}} \wedge w_{\tilde{b}}, u_{\tilde{a}} \vee u_{\tilde{b}}>
\end{aligned}
$$

$$
\begin{gathered}
\tilde{a} \tilde{b}=\left\{\begin{array}{l}
<(\underline{a b}, a b, \bar{a} \bar{b}) ; w_{\tilde{a}} \wedge w_{\tilde{b}}, u_{\tilde{a}} \vee u_{\tilde{b}}>\text { if } \tilde{a}>0 \text { and } \tilde{b}>0 \\
<(a \bar{b}, a b, \bar{a} b) ; w_{\tilde{a}} \wedge w_{\tilde{b}}, u_{\tilde{a}} \vee u_{\tilde{b}}>\text { if } \tilde{a}<0 \text { and } \tilde{b}>0 \\
<(\bar{a} \bar{b}, a b, \underline{a b}) ; w_{\tilde{a}} \wedge w_{\tilde{b}}, u_{\tilde{a}} \vee u_{\tilde{b}}>\text { if } \tilde{a}<0 \text { and } \tilde{b}<0
\end{array}\right. \\
\tilde{a} / \tilde{b}=\left\{\begin{array}{l}
<(\underline{a} / \bar{b}, a / b, \bar{a} / \underline{b}) ; w_{\tilde{a}} \wedge w_{\tilde{b}}, u_{\tilde{a}} \vee u_{\tilde{b}}>\text { if } \tilde{a}>0 \text { and } \tilde{b}>0 \\
<(\bar{a} / \bar{b}, a / b, \underline{a} / \underline{b}) ; w_{\tilde{a}} \wedge w_{\tilde{b}}, u_{\tilde{a}} \vee u_{\tilde{b}}>\text { if } \tilde{a}<0 \text { and } \tilde{b}>0 \\
<(\bar{a} / \underline{b}, a / b, \underline{a} / \bar{b}) ; w_{\tilde{a}} \wedge w_{\tilde{b}}, u_{\tilde{a}} \vee u_{\tilde{b}}>\text { if } \tilde{a}<0 \text { and } \tilde{b}<0
\end{array}\right. \\
\lambda \tilde{a}=\left\{\begin{array}{l}
<(\lambda \underline{a}, \lambda a, \lambda \bar{a}) ; w_{\tilde{a}}, u_{\tilde{a}}>\text { if } \lambda>0 \\
<(\lambda \bar{a}, \lambda a, \lambda \underline{a}) ; w_{\tilde{a}}, u_{\tilde{a}}>\text { if } \lambda<0
\end{array}\right. \\
\tilde{a}^{-1}=<(1 / \bar{a}, 1 / a, 1 / \underline{a}) ; w_{\tilde{a}}, u_{\tilde{a}}>,
\end{gathered}
$$

where the symbols " $\wedge$ " and " $\vee$ " are the min and max operators, respectively.

It is proven that the results from multiplication and division are not TIFNs. But, we often use TIFNs to express these operational results approximately.

Obviously, if $w_{\tilde{a}}=1$ and $u_{\tilde{a}}=0$, i.e., $\tilde{a}=<(\underline{a}, a, \bar{a}) ; 1,0>$ and $\tilde{b}=<(\underline{b}, b, \bar{b}) ; 1,0>$ are TFNs, then Eqs. (4)-(9) degenerate to the arithmetic operations of the TFNs ${ }^{22}$. Hence, the arithmetic operations of TIFNs are a generalization of those of the TFNs ${ }^{22}$.

\subsection{Cut sets of an TIFN}

According to the cut sets of the IF set defined in [10], the cut sets of an TIFN can be defined as follows.

Definition 3. A $(\alpha, \beta)$-cut set of $\tilde{a}=<(\underline{a}, a, \bar{a}) ; w_{\tilde{a}}, u_{\tilde{a}}>$ is a crisp subset of $\mathrm{R}$, which is defined as follows:

$$
\tilde{a}_{\beta}^{\alpha}=\left\{x \mid \mu_{\tilde{a}}(x) \geq \alpha, v_{\tilde{a}}(x) \leq \beta\right\},
$$

where $0 \leq \alpha \leq w_{\tilde{a}}, u_{\tilde{a}} \leq \beta \leq 1$ and $0 \leq \alpha+\beta \leq 1$.

Definition 4. A $\alpha$-cut set of $\tilde{a}=<(\underline{a}, a, \bar{a}) ; w_{\tilde{a}}, u_{\tilde{a}}>$ is $a$ crisp subset of $\mathrm{R}$, which is defined as follows:

$$
\tilde{a}^{\alpha}=\left\{x \mid \mu_{\tilde{a}}(x) \geq \alpha\right\} .
$$

Using Eq. (1) and Definition 4, it follows that $\tilde{a}^{\alpha}$ is a closed interval, denoted by $\tilde{a}^{\alpha}=\left[L^{\alpha}(\tilde{a}), R^{\alpha}(\tilde{a})\right]$, which can be calculated as follows:

$\left[L^{\alpha}(\tilde{a}), R^{\alpha}(\tilde{a})\right]=\left[\underline{a}+\alpha(a-\underline{a}) / w_{\tilde{a}}, \bar{a}-\alpha(\bar{a}-a) / w_{\tilde{a}}\right]$.

The support of the TIFN $\tilde{a}$ for the membership function is defined as follows: 
D.F. Li et al.

$$
\operatorname{supp}_{\mu}(\tilde{a})=\left\{x \mid \mu_{\tilde{a}}(x) \geq 0\right\},
$$

i.e., $\operatorname{supp}_{\mu}(\tilde{a})=\tilde{a}^{0}=[\underline{a}, \bar{a}]$.

Definition 5. A $\beta$-cut set of $\tilde{a}=<(\underline{a}, a, \bar{a}) ; w_{\tilde{a}}, u_{\tilde{a}}>$ is $a$ crisp subset of $\mathrm{R}$, which is defined as follows:

$$
\tilde{a}_{\beta}=\left\{x \mid v_{\tilde{a}}(x) \leq \beta\right\},
$$

where $u_{\tilde{a}} \leq \beta \leq 1$.

Using Eq. (2) and Definition 5, it follows that $\tilde{a}_{\beta}$ is a closed interval, denoted by $\tilde{a}_{\beta}=\left[L_{\beta}(\tilde{a}), R_{\beta}(\tilde{a})\right]$, which can be calculated as follows:

$$
\begin{aligned}
{\left[L_{\beta}(\tilde{a}), R_{\beta}(\tilde{a})\right]=} & {\left[\left[(1-\beta) a+\left(\beta-u_{\tilde{a}}\right) \underline{a}\right] /\left(1-u_{\tilde{a}}\right),[(1-\beta) a+\right.} \\
& \left.\left.\left(\beta-u_{\tilde{a}}\right) \bar{a}\right] /\left(1-u_{\tilde{a}}\right)\right]
\end{aligned}
$$

The support of the TIFN $\tilde{a}$ for the non-membership function is defined as follows:

$$
\operatorname{supp}_{v}(\tilde{a})=\left\{x \mid v_{\tilde{a}}(x) \leq 1\right\},
$$

i.e., $\operatorname{supp}_{v}(\tilde{a})=\tilde{a}_{1}=[\underline{a}, \bar{a}]$.

\section{Characteristics of TIFNs and the Value and Ambiguity based Ranking Method}

\subsection{Value and ambiguity of an TIFN}

In this subsection, the value and ambiguity of an TIFN are defined.

Definition 6. Let $\tilde{a}^{\alpha}$ and $\tilde{a}_{\beta}$ be any $\alpha$-cut set and $\beta$ cut set of an TIFN $\tilde{a}=<(\underline{a}, a, \bar{a}) ; w_{\tilde{a}}, u_{\tilde{a}}>$, respectively. Then the values of the membership function $\mu_{\tilde{a}}$ and the non-membership function $v_{\tilde{a}}$ for the TIFN a are defined as follows:

$$
V_{\mu}(\tilde{a})=\int_{0}^{w_{\tilde{a}}}\left(L^{\alpha}(\tilde{a})+R^{\alpha}(\tilde{a})\right) f(\alpha) \mathrm{d} \alpha
$$

and

$$
V_{v}(\tilde{a})=\int_{u_{\tilde{a}}}^{1}\left(L_{\beta}(\tilde{a})+R_{\beta}(\tilde{a})\right) g(\beta) \mathrm{d} \beta,
$$

respectively.

The function $f(\alpha)=\alpha\left(\alpha \in\left[0, w_{\tilde{a}}\right]\right)$ gives different weights to elements in different $\alpha$-cut sets. In fact, $f(\alpha)$ diminishes the contribution of the lower $\alpha$-cut sets, which is reasonable since these cut sets arising from values of $\mu_{\tilde{a}}$ have a considerable amount of uncertainty. Obviously, $V_{\mu}(\tilde{a})$ synthetically reflects the information on every membership degree, and may be regarded as a central value that represents from the membership function point of view. Similarly, the function $g(\beta)=1-\beta \quad\left(\beta \in\left[u_{\tilde{a}}, 1\right]\right)$ has the effect of weighting on the different $\beta$-cut sets. $g(\beta)$ diminishes the contribution of the higher $\beta$ - cut sets, which is reasonable since these cut sets arising from values of $v_{\tilde{a}}$ have a considerable amount of uncertainty. $V_{v}(\tilde{a})$ synthetically reflects the information on every nonmembership degree and may be regarded as a central value that represents from the non-membership function point of view.

Definition 7. Let $\tilde{a}^{\alpha}$ and $\tilde{a}_{\beta}$ be $\alpha$-cut set and $\beta$-cut set of an TIFN $\tilde{a}=<(\underline{a}, a, \bar{a}) ; w_{\tilde{a}}, u_{\tilde{a}}>$, respectively. Then the ambiguities of the membership function $\mu_{\tilde{a}}$ and the non-membership function $v_{\tilde{a}}$ for the TIFN $\tilde{a}$ are defined as follows:

$$
A_{\mu}(\tilde{a})=\int_{0}^{w_{\tilde{a}}}\left(R^{\alpha}(\tilde{a})-L^{\alpha}(\tilde{a})\right) f(\alpha) \mathrm{d} \alpha
$$

and

$$
A_{v}(\tilde{a})=\int_{u_{\tilde{a}}}^{1}\left(R_{\beta}(\tilde{a})-L_{\beta}(\tilde{a})\right) g(\beta) \mathrm{d} \beta,
$$

respectively.

It is easy to see that $R^{\alpha}(\tilde{a})-L^{\alpha}(\tilde{a})$ and $R_{\beta}(\tilde{a})-L_{\beta}(\tilde{a})$ are just about the lengths of the intervals $\tilde{a}^{\alpha}$ and $\tilde{a}_{\beta}$, respectively. Thus, $A_{\mu}(\tilde{a})$ and $A_{v}(\tilde{a})$ may be regarded as the "global spreads" of the membership function $\mu_{\tilde{a}}$ and the non-membership function $v_{\tilde{a}}$. Obviously, $A_{\mu}(\tilde{a})$ and $A_{v}(\tilde{a})$ basically measure how much there is vagueness in the TIFN $\tilde{a}$.

The values of the membership function and the nonmembership function of the TIFN $\tilde{a}$ are calculated as follows:

$$
V_{\mu}(\tilde{a})=(\underline{a}+4 a+\bar{a}) w_{\tilde{a}}^{2} / 6
$$

and

$$
V_{v}(\tilde{a})=(\underline{a}+4 a+\bar{a})\left(1-u_{\tilde{a}}\right)^{2} / 6,
$$

respectively.

The ambiguities of the membership function and the non-membership function of the TIFN $\tilde{a}$ are calculated as follows:

$$
A_{\mu}(\tilde{a})=(\bar{a}-\underline{a}) w_{\tilde{a}}^{2} / 6
$$


and

$$
A_{v}(\tilde{a})=(\bar{a}-\underline{a})\left(1-u_{\tilde{a}}\right)^{2} / 6,
$$

respectively.

\subsection{The value and ambiguity based ranking method}

Based on the above value and ambiguity of an TIFN, a new ranking method of TIFNs is proposed in this subsection. A value-index and an ambiguity-index for $\tilde{a}$ are firstly defined as follows.

Definition 8. Let $\tilde{a}=<(\underline{a}, a, \bar{a}) ; w_{\tilde{a}}, u_{\tilde{a}}>$ be an TIFN. A value-index and an ambiguity-index for $\tilde{a}$ are defined as follows:

$$
V_{\lambda}(\tilde{a})=\lambda V_{\mu}(\tilde{a})+(1-\lambda) V_{v}(\tilde{a})
$$

and

$$
A_{\lambda}(\tilde{a})=\lambda A_{\mu}(\tilde{a})+(1-\lambda) A_{v}(\tilde{a}),
$$

respectively, where $\lambda \in[0,1]$ is a weight which represents the decision maker's preference information.

$\lambda \in(1 / 2,1]$ shows that decision maker prefers to certainty or positive feeling; $\lambda \in[0,1 / 2)$ shows that decision maker prefers to uncertainty or negative feeling; $\lambda=1 / 2$ shows that decision maker is indifferent to between certainty and uncertainty. Therefore, the valueindex and the ambiguity-index may reflect the decision maker's subjectivity attitudes to the TIFN.

Let $\tilde{a}=<(\underline{a}, a, \bar{a}) ; w_{\tilde{a}}, u_{\tilde{a}}>$ and $\tilde{b}=<(\underline{b}, b, \bar{b}) ; w_{\tilde{b}}, u_{\tilde{b}}>$ be two TIFNs. A lexicographic ranking procedure based on the value-index and ambiguity-index can be summarized as follows:

Step 1 Compare $V_{\lambda}(\tilde{a})$ and $V_{\lambda}(\tilde{b})$ for a given weight $\lambda$. If they are equal, then go to the step 2. Otherwise, rank $\tilde{a}$ and $\tilde{b}$ according to the relative positions of $V_{\lambda}(\tilde{a})$ and $V_{\lambda}(\tilde{b})$. Namely, if $V_{\lambda}(\tilde{a})>V_{\lambda}(\tilde{b})$, then $\tilde{a}$ is greater than $\tilde{b}$, denoted by $\tilde{a}>\tilde{b}$; if $V_{\lambda}(\tilde{a})<V_{\lambda}(\tilde{b})$, then $\tilde{a}$ is smaller than $\tilde{b}$, denoted by $\tilde{a}<\tilde{b}$.

Step 2 Compare $A_{\lambda}(\tilde{a})$ and $A_{\lambda}(\tilde{b})$ for the same given $\lambda$. If they are equal, then $\tilde{a}$ and $\tilde{b}$ are equal. Otherwise, rank $\tilde{a}$ and $\tilde{b}$ according to the relative positions of $-A_{\lambda}(\tilde{a})$ and $-A_{\lambda}(\tilde{b})$. Namely, if $-A_{\lambda}(\tilde{a})>-A_{\lambda}(\tilde{b})$, then $\tilde{a}>\tilde{b}$; if $-A_{\lambda}(\tilde{a})<-A_{\lambda}(\tilde{b})$, then $\tilde{a}<\tilde{b}$.

Wang and Kerre ${ }^{24}$ proposed some axioms which are used to evaluate the rationality of a ranking method of fuzzy numbers. It is easy to verify that $V_{\lambda}(\tilde{a})$ satisfies the axioms $A_{1}-A_{6}{ }^{24}$. Proofs that $V_{\lambda}(\tilde{a})$ satisfies the axioms $A_{1}-A_{3}$ and $A_{5}$ are easily completed. In the following, we focus on verifying that $V_{\lambda}(\tilde{a})$ satisfies the axioms $A_{4}$ and $A_{6}{ }^{24}$.

Theorem 1. Let $\tilde{a}=<(\underline{a}, a, \bar{a}) ; w_{\tilde{a}}, u_{\tilde{a}}>\quad$ and $\tilde{b}=<(\underline{b}, b, \bar{b}) ; w_{\tilde{b}}, u_{\tilde{b}}>$ be two TIFNs with $w_{\tilde{a}}=w_{\tilde{b}}$ and $u_{\tilde{a}}=u_{\tilde{b}}$. If $\underline{a}>\bar{b}$, then $\tilde{a}>\tilde{b}$.

Proof. It is derived from Eq. (27) that

$$
V_{\mu}(\tilde{a})=\int_{0}^{w_{\tilde{a}}}\left(L^{\alpha}(\tilde{a})+R^{\alpha}(\tilde{a})\right) \alpha \mathrm{d} \alpha \geq \int_{0}^{w_{\bar{a}}} 2 \underline{a} \alpha \alpha \mathrm{d} \alpha=\underline{a} w_{\tilde{a}}^{2}
$$

and

$$
V_{\mu}(\tilde{b})=\int_{0}^{w_{\tilde{b}}}\left(L^{\alpha}(\tilde{b})+R^{\alpha}(\tilde{b})\right) \alpha \mathrm{d} \alpha \leq \int_{0}^{w_{\tilde{b}}} 2 \bar{b} \alpha \mathrm{d} \alpha=\bar{b} w_{\tilde{b}}^{2} .
$$

Combining with both $\underline{a}>\bar{b}$ and $w_{\tilde{a}}=w_{\tilde{b}}$, it directly follows that $V_{\mu}(\tilde{a})>V_{\mu}(\tilde{b})$.

Similarly, it follows that

$$
\begin{aligned}
V_{v}(\tilde{a}) & =\int_{u_{\tilde{a}}}^{1}\left(L_{\beta}(\tilde{a})+R_{\beta}(\tilde{a})\right)(1-\beta) \mathrm{d} \beta \\
& \geq \int_{u_{\tilde{a}}}^{1} 2 \underline{a}(1-\beta) \mathrm{d} \beta=\underline{a}\left(1-u_{\tilde{a}}\right)^{2}
\end{aligned}
$$

and

$$
\begin{aligned}
V_{v}(\tilde{b}) & =\int_{u_{\tilde{b}}}^{1}\left(L_{\beta}(\tilde{b})+R_{\beta}(\tilde{b})\right)(1-\beta) \mathrm{d} \beta \\
& \leq \int_{u_{\tilde{b}}}^{1} 2 \bar{b}(1-\beta) \mathrm{d} \beta=\bar{b}\left(1-u_{\tilde{b}}\right)^{2}
\end{aligned}
$$

respectively. Combining with both $\underline{a}>\bar{b}$ and $u_{\tilde{a}}=u_{\tilde{b}}$, then $V_{v}(\tilde{a})>V_{v}(\tilde{b})$. Therefore,

$$
\lambda V_{\mu}(\tilde{a})+(1-\lambda) V_{v}(\tilde{a})>\lambda V_{\mu}(\tilde{b})+(1-\lambda) V_{v}(\tilde{b}),
$$

i.e., $V_{\lambda}(\tilde{a})>V_{\lambda}(\tilde{b})$. Hence, $\tilde{a}>\tilde{b}$.

Theorem 2. Let $\tilde{a}=<(\underline{a}, a, \bar{a}) ; w_{\tilde{a}}, u_{\tilde{a}}>$, $\tilde{b}=<(\underline{b}, b, \bar{b}) ; w_{\tilde{b}}, u_{\tilde{b}}>$ and $\tilde{c}=<(\underline{c}, c, \bar{c}) ; w_{\tilde{c}}, u_{\tilde{c}}>\quad b e$ TIFNs with $w_{\tilde{a}}=w_{\tilde{b}}$ and $u_{\tilde{a}}=u_{\tilde{b}}$. If $\tilde{a}>\tilde{b}$, then $\tilde{a}+\tilde{c}>\tilde{b}+\tilde{c}$.

Proof. It is derived from Eq. (27) that

$$
\begin{aligned}
V_{\mu}(\tilde{a}+\tilde{c}) & =\int_{0}^{w_{\tilde{a}} \wedge w_{\tilde{c}}}\left(L^{\alpha}(\tilde{a})+R^{\alpha}(\tilde{a})+L^{\alpha}(\tilde{c})+R^{\alpha}(\tilde{c})\right) \alpha \mathrm{d} \alpha \\
& =\int_{0}^{w_{\bar{a}} \wedge w_{\bar{c}}}\left(L^{\alpha}(\tilde{a})+R^{\alpha}(\tilde{a})\right) \alpha \mathrm{d} \alpha+\int_{0}^{w_{\tilde{a}} \wedge w_{\tilde{c}}}\left(L^{\alpha}(\tilde{c})+R^{\alpha}(\tilde{c})\right) \alpha \mathrm{d} \alpha
\end{aligned}
$$

and 


$$
\begin{aligned}
V_{\mu}(\tilde{b}+\tilde{c}) & =\int_{0}^{w_{\bar{b}} \wedge w_{\bar{c}}}\left(L^{\alpha}(\tilde{b})+R^{\alpha}(\tilde{b})+L^{\alpha}(\tilde{c})+R^{\alpha}(\tilde{c})\right) \alpha \mathrm{d} \alpha \\
& =\int_{0}^{w_{\tilde{b}} \wedge w_{\tilde{c}}}\left(L^{\alpha}(\tilde{b})+R^{\alpha}(\tilde{b})\right) \alpha \mathrm{d} \alpha+\int_{0}^{w_{\tilde{b}} \wedge w_{\tilde{c}}}\left(L^{\alpha}(\tilde{c})+R^{\alpha}(\tilde{c})\right) \alpha \mathrm{d} \alpha
\end{aligned}
$$

respectively. Because of $\tilde{a}>\tilde{b}$ and $w_{\tilde{a}}=w_{\tilde{b}}$, it follows that

$$
\int_{0}^{w_{\hat{a}} \wedge w_{\tilde{c}}}\left(L^{\alpha}(\tilde{a})+R^{\alpha}(\tilde{a})\right) \alpha \mathrm{d} \alpha>\int_{0}^{w_{\bar{b}} \wedge w_{\tilde{c}}}\left(L^{\alpha}(\tilde{b})+R^{\alpha}(\tilde{b})\right) \alpha \mathrm{d} \alpha .
$$

Hence, $V_{\mu}(\tilde{a}+\tilde{c})>V_{\mu}(\tilde{b}+\tilde{c})$.

Similarly, it follows that

$$
\begin{aligned}
V_{v}(\tilde{a}+\tilde{c}) & =\int_{u_{\bar{a}} v_{u_{\bar{c}}}}^{1}\left(L_{\beta}(\tilde{a})+R_{\beta}(\tilde{a})+L_{\beta}(\tilde{c})+R_{\beta}(\tilde{c})\right)(1-\beta) \mathrm{d} \beta \\
& =\int_{u_{\tilde{a}} v_{\tilde{c}}}^{1}\left(L_{\beta}(\tilde{a})+R_{\beta}(\tilde{a})\right)(1-\beta) \mathrm{d} \beta+\int_{u_{\tilde{a}} \backslash v_{\bar{c}}}^{1}\left(L_{\beta}(\tilde{c})+R_{\beta}(\tilde{c})\right)(1-\beta) \mathrm{d} \beta
\end{aligned}
$$

and

$$
\begin{aligned}
V_{v}(\tilde{b}+\tilde{c}) & =\int_{u_{\tilde{b}} \vee u_{\tilde{c}}}^{1}\left(L_{\beta}(\tilde{b})+R_{\beta}(\tilde{b})+L_{\beta}(\tilde{c})+R_{\beta}(\tilde{c})\right)(1-\beta) \mathrm{d} \beta \\
& =\int_{u_{\tilde{b}} \vee u_{\tilde{c}}}^{1}\left(L_{\beta}(\tilde{b})+R_{\beta}(\tilde{b})\right)(1-\beta) \mathrm{d} \beta+\int_{u_{\tilde{b}} \backslash u_{\tilde{c}}}^{1}\left(L_{\beta}(\tilde{c})+R_{\beta}(\tilde{c})\right)(1-\beta) \mathrm{d} \beta
\end{aligned}
$$

respectively.

It is readily derived from $\tilde{a}>\tilde{b}$ and $u_{\tilde{a}}=u_{\tilde{b}}$ that

$$
\int_{u_{\tilde{a}} \vee u_{\bar{c}}}^{1}\left(L_{\beta}(\tilde{a})+R_{\beta}(\tilde{a})\right)(1-\beta) \mathrm{d} \beta>\int_{u_{\bar{b}} \vee v_{\tilde{c}}}^{1}\left(L_{\beta}(\tilde{b})+R_{\beta}(\tilde{b})\right)(1-\beta) \mathrm{d} \beta .
$$

Hence, $V_{v}(\tilde{a}+\tilde{c})>V_{v}(\tilde{b}+\tilde{c})$.Then,

$\lambda V_{\mu}(\tilde{a}+\tilde{c})+(1-\lambda) V_{v}(\tilde{a}+\tilde{c})>\lambda V_{\mu}(\tilde{b}+\tilde{c})+(1-\lambda) V_{v}(\tilde{b}+\tilde{c})$,

i.e., $V_{\lambda}(\tilde{a}+\tilde{c})>V_{\lambda}(\tilde{b}+\tilde{c})$. Therefore, $\tilde{a}+\tilde{c}>\tilde{b}+\tilde{c}$.

\section{An Extended MADM Method based on the Value and Ambiguity based Ranking Procedure}

In this section, we will apply the above ranking method of TIFNs to solve MADM problems in which the ratings of alternatives on attributes are expressed using TIFNs. Sometimes such MADM problems are called as MADM problems with TIFNs for short. Suppose that there exists an alternative set $A=\left\{A_{1}, A_{2}, \cdots, A_{m}\right\}$, which consists of $m$ non-inferior alternatives from which the most preferred alternative has to be selected. Each alternative is assessed on $n$ attributes. Denote the set of all attributes by $X=\left\{X_{1}, X_{2}, \cdots, X_{n}\right\}$. Assume that ratings of alternatives on attributes are given using TIFNs. Namely, the rating of any alternative $A_{i} \in A$ $(i=1,2, \cdots, m)$ on each attribute $X_{j} \in X(j=1,2, \cdots, n)$ is an TIFN $\tilde{a}_{i j}=<\left(\underline{a}_{i j}, a_{i j}, \bar{a}_{i j}\right) ; w_{\tilde{a}_{i j}}, u_{\tilde{a}_{i j}}>$. Thus an MADM problem with TIFNs can be expressed concisely in the matrix format as $\left(\tilde{a}_{i j}\right)_{m \times n}$.
Due to the fact that different attributes may have different importance. Assume that the relative weight of the attribute $X_{j}$ is $\omega_{j}(j=1,2, \cdots, n)$, satisfying the normalization conditions: $\omega_{j} \in[0,1]$ and $\sum_{j=1}^{n} \omega_{j}=1$. Let $\boldsymbol{\omega}=\left(\omega_{1}, \omega_{2}, \cdots, \omega_{n}\right)^{\mathrm{T}}$ be the relative weight vector of all attributes.

The extended additive weighted method for the MADM problem with TIFNs can be summarized as follows:

(i) Normalize the TIFN decision matrix. In order to eliminate the effect of different physical dimensions on the final decision making results, the normalized TIFN decision matrix can be calculated using the following formulae:

$$
\tilde{r}_{i j}=<\left(\frac{\underline{a}_{i j}}{\bar{a}_{j}^{+}}, \frac{a_{i j}}{\bar{a}_{j}^{+}}, \frac{\bar{a}_{i j}}{\bar{a}_{j}^{+}}\right) ; w_{\tilde{a}_{i j}}, u_{\tilde{a}_{i j}}>(i=1,2, \cdots, m ; j \in B)
$$

and

$$
\tilde{r}_{i j}=<\left(\frac{\underline{a}_{j}^{-}}{\bar{a}_{i j}}, \frac{\underline{a}_{j}^{-}}{a_{i j}}, \frac{\underline{a}_{j}^{-}}{\underline{a}_{i j}}\right) ; w_{\tilde{a}_{i j}}, u_{\tilde{a}_{i j}}>(i=1,2, \cdots, m j \in C),
$$

respectively, where $B$ and $C$ are the subscript sets of benefit attributes and cost attributes, and

$$
\bar{a}_{j}^{+}=\max \left\{\bar{a}_{i j} \mid i=1,2, \cdots, m\right\} \quad(j \in B)
$$

and

$$
\underline{a}_{j}^{-}=\min \left\{\underline{a}_{i j} \mid i=1,2, \cdots, m\right\} \quad(j \in C) .
$$

(ii) Construct the weighted normalized TIFN decision matrix. Using Eq. (8), the weighted normalized TIFN decision matrix can be calculated as $\left(\tilde{u}_{i j}\right)_{m \times n}$, where

$$
\tilde{u}_{i j}=\omega_{j} \tilde{r}_{i j} .
$$

(iii) Calculate the weighted comprehensive values of alternatives. Using Eq. (4), the weighted comprehensive values of alternatives $A_{i}(i=1,2, \cdots, m)$ are calculated as follows:

$$
\tilde{S}_{i}=\sum_{j=1}^{n} \tilde{u}_{i j},
$$

respectively. Obviously, $\tilde{S}_{i}(i=1,2, \cdots, m)$ are TIFNs.

(iv) Rank all alternatives. The ranking order of the alternatives $A_{i}$ can be generated according to the nonincreasing order of the TIFNs $\tilde{S}_{i}(i=1,2, \cdots, m)$ by using the value and ambiguity based ranking method proposed in Section 3. 


\section{A Numerical Example}

We analyze a personnel selection problem. Suppose that a software company desires to hire a system analyst. After preliminary screening, three candidates $A_{1}, A_{2}$ and $A_{3}$ remain for further evaluation. The decision making committee assesses the three candidates based on five attributes, including emotional steadiness $\left(X_{1}\right)$, oral communication skill $\left(X_{2}\right)$, personality ( $\left.X_{3}\right)$, past experience $\left(X_{4}\right)$ and self-confidence $\left(X_{5}\right)$. Assume that the total mark of each attribute is 10. Using statistical methods, the ratings of the candidates with respect to the attributes are given as in Table 1 , where $<(5.7,7.7,9.3) ; 0.7,0.2>$ in the Table 1 is an TIFN which indicates that the mark of the candidate $A_{1}$ with respect to the attribute $X_{1}$ is about 7.7 with the maximum satisfaction degree is 0.7 , while the minimum nonsatisfaction degree is 0.2 . In other words, the hesitation degree is 0.1 . Other TIFNs in Table 1 are explained similarly.

Since the five attributes are benefit attributes, according to Eqs. (27) and (29), the weighted normalized TIFN decision matrix is obtained as in Table 2.

Table 1. The TIFN decision matrix

\begin{tabular}{cccclc}
\hline \multirow{2}{*}{ Alternatives } & \multicolumn{5}{c}{ Attributes } \\
& \multicolumn{1}{c}{$X_{1}$} & \multicolumn{1}{c}{$X_{2}$} & $X_{3}$ & \multicolumn{1}{c}{$X_{4}$} & $X_{5}$ \\
\hline$A_{1}$ & $<(5.7,7.7,9.3) ; 0.7,0.2>$ & $<(5,7,9) ; 0.6,0.3>$ & $<(5.7,7.7,9) ; 0.8,0.1>$ & $<(8.33,9.67,10) ; 0.6,0.4>$ & $<(3,5,7) ; 0.6,0.3>$ \\
$A_{2}$ & $<(6.5,8.6,10) ; 0.4,0.5>$ & $<(8,9,10) ; 0.6,0.3>$ & $<(8.3,9.7,10) ; 0.7,0.2>$ & $<(8,9,10) ; 0.6,0.3>$ & $<(7,9,10) ; 0.6,0.2>$ \\
$A_{3}$ & $<(6.5,8.2,9.3) ; 0.8,0.1>$ & $<(7,9,10) ; 0.7,0.2>$ & $<(0,9,10) ; 0.5,0.2>$ & $<(6,8,9) ; 0.6,0.2>$ & $<(6.3,8.3,9.7) ; 0.7,0.2>$ \\
\hline
\end{tabular}

Table 2. The weighted normalized TIFN decision matrix

\begin{tabular}{|c|c|c|c|c|c|}
\hline \multirow{2}{*}{ Alternatives } & \multicolumn{5}{|c|}{ Attributes } \\
\hline & $X_{1}$ & $X_{2}$ & $X_{3}$ & $X_{4}$ & $X_{5}$ \\
\hline$A_{1}$ & $<(0.083,0.111,0134) ; 0.7,0.2>$ & $<(0.15,0.21,0.27) ; 0.6,0.3>$ & $<(0.068,0.092,0.108) ; 0.8,0.1>$ & $<(0.249,0.291,0.3) ; 0.6,0.4>$ & $<(0.042,0.07,0.098) ; 0.6,0.3>$ \\
\hline$A_{2}$ & $<(0.091,0.12,0.14) ; 0.4,0.5>$ & $<(0.24,0.27,0.3) ; 0.6,0.3>$ & $<(0.1,0.116,0.12) ; 0.7,0.2>$ & $<(0.24,0.27,0.3) ; 0.6,0.3>$ & $<(0.098,0.126,0.14) ; 0.6,0.2>$ \\
\hline$A_{3}$ & $<(0.091,0.115,0.13) ; 0.8,0.1>$ & $<(0.21,0.27,0.3) ; 0.7,0.2>$ & $<(0.084,0.108,0.12) ; 0.5,0.2>$ & $<(0.18,0.24,0.27) ; 0.6,0.2>$ & $<(0.088,0.116,0.136) ; 0.7,0.2>$ \\
\hline
\end{tabular}

Using Eq. (30), the weighted comprehensive values of the candidates $A_{i}(i=1,2,3)$ can be obtained as follows:

$$
\begin{gathered}
\tilde{S}_{1}=<(0.592,0.774,0.910) ; 0.6,0.4>, \\
\tilde{S}_{2}=<(0.769,0.903,1) ; 0.4,0.5>
\end{gathered}
$$

and

$$
\tilde{S}_{3}=<(0.653,0.849,0.956) ; 0.5,0.2>,
$$

respectively.

According to Eqs. (21) and (22), the values of membership functions and non-membership functions of $\tilde{S}_{1}, \tilde{S}_{2}$ and $\tilde{S}_{3}$ can be calculated as follows:

$$
\begin{array}{ll}
V_{\mu}\left(\tilde{S}_{1}\right)=0.276, & V_{\nu}\left(\tilde{S}_{1}\right)=0.276, \\
V_{\mu}\left(\tilde{S}_{2}\right)=0.144, & V_{v}\left(\tilde{S}_{2}\right)=0.224
\end{array}
$$

and

$$
V_{\mu}\left(\tilde{S}_{3}\right)=0.209, \quad V_{v}\left(\tilde{S}_{3}\right)=0.534,
$$

respectively.
Using Eq. (25), the value-indices of $\tilde{S}_{1}, \tilde{S}_{2}$ and $\tilde{S}_{3}$ can be obtained as follows:

$$
\begin{gathered}
V_{\lambda}\left(\tilde{S}_{1}\right)=0.276, \\
V_{\lambda}\left(\tilde{S}_{2}\right)=0.144 \lambda+0.224(1-\lambda)
\end{gathered}
$$

and

$$
V_{\lambda}\left(\tilde{S}_{3}\right)=0.209 \lambda+0.534(1-\lambda),
$$

respectively, depicted as in Fig.2.

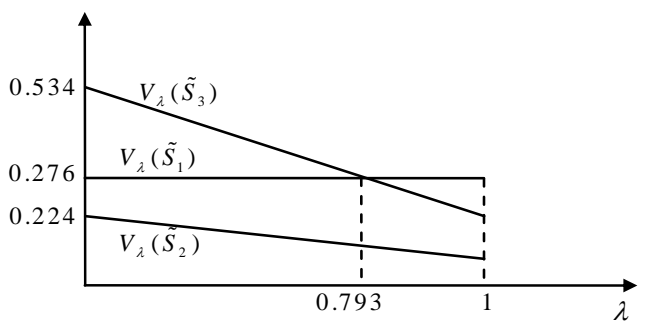

Fig.2. The value-indices of $\tilde{S}_{1}, \tilde{S}_{2}$ and $\tilde{S}_{3}$ 
From Fig.2, it is easily seen that the value-indices of $\tilde{S}_{3}$ and $\tilde{S}_{1}$ are equal when $\lambda=0.793$, i.e., $V_{0.793}\left(\tilde{S}_{3}\right)=V_{0.793}\left(\tilde{S}_{1}\right)=0.276$. According to Eqs. (23), (24) and (26), the ambiguity-indices of $\tilde{S}_{3}$ and $\tilde{S}_{1}$ can be calculated as $A_{0.793}\left(\tilde{S}_{1}\right)=0.019$ and $A_{0.793}\left(\tilde{S}_{3}\right)=0.017$, respectively. Therefore, the ranking order of $\tilde{S}_{3}$ and $\tilde{S}_{1}$ is $\tilde{S}_{3}>\tilde{S}_{1}$.

It is easy to see from Fig.2 that $V_{\lambda}\left(\tilde{S}_{3}\right)>V_{\lambda}\left(\tilde{S}_{1}\right)>V_{\lambda}\left(\tilde{S}_{2}\right)$ for any given weight $\lambda \in[0,0.793]$. Hence, the ranking order of the three candidates is $A_{3} \succ A_{1} \succ A_{2}$ if $\lambda \in[0,0.793]$. In this case, the best selection is the candidate $A_{3}$. However, if $\lambda \in(0.793,1]$ then $V_{\lambda}\left(\tilde{S}_{1}\right)>V_{\lambda}\left(\tilde{S}_{3}\right)>V_{\lambda}\left(\tilde{S}_{2}\right)$, and the ranking order of the three candidates is $A_{1} \succ A_{3} \succ A_{2}$, and the best selection is the candidate $A_{1}$.

If we do not consider the maximum degrees of membership and the minimum degrees of nonmembership, i.e., assume that $w_{\tilde{a}_{i j}}=1$ and $u_{\tilde{a}_{i j}}=0$, then the TIFNs in Table 1 (i.e., ratings of the alternatives on the attributes) reduce to TFNs, denoted by $\hat{a}=\left(\underline{a}_{i j}, a_{i j}, \bar{a}_{i j}\right)$. Thus, the above MADM problem with TIFNs reduces to the MADM problem with TFNs. Using the similar weighted average method for the MADM problems, the weighted comprehensive values of the candidates $A_{i}(i=1,2,3)$ can be obtained as follows:

$$
\begin{gathered}
\hat{S}_{1}=(0.592,0.774,0.910), \\
\hat{S}_{2}=(0.769,0.903,1)
\end{gathered}
$$

and

$$
\hat{S}_{3}=(0.653,0.849,0.956)
$$

respectively.

Obviously, $\hat{S}_{i}(i=1,2,3)$ are TFNs. Using the existing ranking methods of fuzzy numbers, It is not difficult to see that the ranking order is $\hat{S}_{2}>\hat{S}_{3}>\hat{S}_{1}$, which is conflicting with the obtained result above. This analysis result shows that the maximum degrees of membership and the minimum degrees of nonmembership play an important role in the ranking order of TIFNs. Intuitively, it is perhaps more reasonable to choose $\tilde{S}_{3}=<(0.653,0.849,0.956) ; 0.5,0.2>$ instead of $\tilde{S}_{2}=<(0.769,0.903,1) ; 0.4,0.5>$ for a pessimistic decision maker in that $\tilde{S}_{3}$ has larger membership degree and smaller non-membership degree than $\tilde{S}_{2}$.
In the following, we apply Wang and Zhang's method ${ }^{15}$ to rank the TIFNs $\tilde{S}_{1}, \tilde{S}_{2}$ and $\tilde{S}_{3}$. The expected value intervals of $\tilde{S}_{1}, \tilde{S}_{2}$ and $\tilde{S}_{3}$ can be calculated as follows:

$$
\begin{gathered}
I_{\gamma}\left(\tilde{S}_{1}\right)=0.41+0.095 \gamma, \\
I_{\gamma}\left(\tilde{S}_{2}\right)=[0.334+0.046 \gamma, 0.418+0.058 \gamma]
\end{gathered}
$$

and

$$
I_{\gamma}\left(\tilde{S}_{3}\right)=[0.376+0.076 \gamma, 0.601+0.121 \gamma],
$$

respectively. Using Eqs. (5) and (19) introduced in [19], for some given specific values $\gamma \in[0,1]$, the ranking orders of $\tilde{S}_{1}, \tilde{S}_{2}$ and $\tilde{S}_{3}$ are obtained as in Table 3 .

Table 3. The ranking results obtained by Wang and

\begin{tabular}{ccccc}
\multicolumn{5}{c}{ Zhang' method ${ }^{15}$} \\
\hline$\gamma$ & $\tilde{S}_{1}$ & $\tilde{S}_{2}$ & $\tilde{S}_{3}$ & ranking results \\
\hline$\gamma=0.1$ & 0.35 & 0.2 & 0.45 & $\tilde{S}_{3}>\tilde{S}_{1}>\tilde{S}_{2}$ \\
$\gamma=0.3$ & 0.36 & 0.19 & 0.45 & $\tilde{S}_{3}>\tilde{S}_{1}>\tilde{S}_{2}$ \\
$\gamma=0.5$ & 0.36 & 0.18 & 0.45 & $\tilde{S}_{3}>\tilde{S}_{1}>\tilde{S}_{2}$ \\
$\gamma=0.793$ & 0.37 & 0.18 & 0.46 & $\tilde{S}_{3}>\tilde{S}_{1}>\tilde{S}_{2}$ \\
\hline
\end{tabular}

From Table 3 , if $\lambda \in[0,0.793]$, then the ranking results obtained by the proposed method are the same as those obtained by Wang and Zhang's method. This shows that the proposed method is effective. However, since $\tilde{S}_{1}=<(0.592,0.774,0.910) ; 0.6,0.4>$ has larger membership degree and non-membership degree than $\tilde{S}_{3}=<(0.653,0.849,0.956) ; 0.5,0.2>$, the decision makers with different preference attitudes may have different choices. Namely, a risk-prone decision maker may prefer $\tilde{S}_{1}$ whereas a risk-averse decision maker may prefer $\tilde{S}_{3}$. These factors cannot be reflected in Wang and Zhang's method ${ }^{15}$. Thus, the proposed method is more reasonable. On the other hand, Wang and Zhang's method ${ }^{15}$ transformed the ranking of TIFNs into that of interval numbers. The ranking of interval numbers is still difficult. However, the proposed method can transform the ranking of TIFNs to that of real numbers. Therefore, the proposed method is easy to be implemented.

\section{Conclusion}

This paper discusses two characteristics of an TIFN, i.e., the value and ambiguity, which are used to define the value-index and ambiguity-index of the TIFN. Then, the 
value-index and ambiguity-index based ranking method is developed for TIFNs. Furthermore, the proposed ranking method is applied to solve MADM problems with TIFNs. The proposed ranking method is easily implemented and has a natural interpretation. It is easily seen that the proposed ranking method can be extended to more general IFNs in a straightforward manner. Due to the fact that an TIFN is a generalization of an TFN, the other existing ranking methods of fuzzy numbers may be extended to TIFNs. More effective ranking methods of TIFNs will be investigated in the near future.

\section{Acknowledgements}

The authors would like to thank the valuable reviews and also appreciate the constructive suggestions from the anonymous referees. This research was sponsored by the Natural Science Foundation of China (No. 70571086, No. 70871117) and the Renwen Foundation of Dalian University of Technology (No. 2008407).

\section{References}

1. S. Abbasbandy and M. Amirfakhrian, The nearest trapezoidal form of a generalized left right fuzzy number, International Journal of Approximate Reasoning 43 (2006) 166-178.

2. S. Abbasbandy and B. Asady, Ranking of fuzzy numbers by sign distance, Information Sciences 176 (2006) 24052416.

3. S. Abbasbandy and T. Hajjari, A new approach for ranking of trapezoidal fuzzy number, Computers and Mathematics with Applications 57 (2009) 413-419.

4. S. J Chen and C. L Hwang, Fuzzy Multiple Attribute Decision Making (Spring, Berlin, 1992).

5. T. Chu and C. Tsao, Ranking fuzzy numbers with an area between the centroid point and original point, Computers and Mathematics with Applications 43 (2002) 11-117.

6. C. Kahraman and A. C. Tolga, An alternative ranking approach and its usage in multi-criteria decision-making, International Journal of Computational Intelligence Systems 2 (2009) 219-235.

7. M. Delgado, M. A Vila and W. Voxman, On a canonical representation of fuzzy numbers, Fuzzy Sets and Systems 93 (1998) 125-135.

8. M. Ma, A. Kandel and M. Friedman, A new approach for defuzzification, Fuzzy Sets and Systems 111 (2000) 351356.

9. R. K. Brouwer, Clustering feature vectors with mixed numerical and categorical attributes, International Journal of Computational Intelligence Systems 1 (2008) 285-298.

10. K. T. Atanassov, Intuitionistic Fuzzy Sets (Springer Heidelberg, 1999).
11. L. A. Zadeh, Fuzzy sets, Information and Control 8 (1965) 338-356.

12. H. B. Mitchell, Ranking intuitionistic fuzzy numbers, International Journal of Uncertainty, Fuzziness and Knowledge Based Systems 12 (2004) 377-386.

13. V. G. Nayagam, G. Venkateshwari and G. Sivaraman, Ranking of intuitionistic fuzzy numbers, IEEE International Conference on Fuzzy Systems (2006)19711974.

14. M. H. Shu, C. H. Cheng and J. R. Chang, Using intuitionistic fuzzy sets for fault-tree analysis on printed circuit board assembly, Microelectronics Reliability 46 (2006) 2139-2148.

15. J. Q. Wang and Z. Zhang, Multi-criteria decisionmaking method with incomplete certain information based on intuitionistic fuzzy number, Control and Decision 24 (2009) 226-230 (in Chinese).

16. J. X. Nan, D. F. Li and M. J. Zhang, A lexicographic method for matrix games with payoffs of triangular intuitionistic fuzzy numbers, International Journal of Computational Intelligence Systems, to appear.

17. D. F. Li and J. B. Yang, Fuzzy linear programming technique for multiattribute group decision making in fuzzy environments, Information Sciences 158 (2004) 263-275.

18. X. B. Li, D. Ruan, J. Liu and Y. Xu, A LinguisticValued Weighted Aggregation Operator to Multiple Attribute Group Decision Making with Quantitative and Qualitative Information, International Journal of Computational Intelligence Systems 3 (2008) 274-284.

19. D. F. Li, Y. C. Wang, S. Liu and F. Shan, Fractional programming methodology for multi-attribute group decision-making using IFS, Applied Soft Computing 9 (2009) 219-225.

20. D. F. Li, Linear programming method for MADM with interval-valued intuitionistic fuzzy sets, Expert Systems with Applications, Available online 13 February 2010.

21. D. F. Li, G. H. Chen and Z. G. Huang, Linear programming method for multiattribute group decision making using IF sets, Information Sciences 180 (9)(2010) 1591-1609.

22. D. Dubois and H. Prade, Fuzzy Sets and Systems: Theory and Applications (Academic Press, New York, 1980).

23. D. F. Li, A note on using intuitionistic fuzzy sets for fault-tree analysis on printed circuit board assembly, Microelectronics Reliability 48 (2008) 1741.

24. X. Wang and E. E. Kerre, Reasonable properties for the ordering of fuzzy quantities (I), Fuzzy Sets and Systems 118 (2001) 375-385. 